\title{
Research on Evaluation Model of the Urban Economy Based on Rough Set and Neural Network
}

\author{
L. Yan \\ Department of Busines s \\ Guilin Institute of Tourism \\ China
}

\begin{abstract}
For the shortage of the traditional evaluation methods of the urban economy, A new evaluation model proposed, establishing an index system of evaluation and decision table on city economy, and extracting important properties of input data from Rough Set and neural network, and evaluation indexes reduced as input of neural network to realize the city's economic strength evaluation, then, using programming with MATLAB and realizing the simulation of rough set and neural network evaluation model, at the same time, consistenting with traditional network model results.
\end{abstract}

Keywords-rough set; neural network; urban economy; MATLAB; evaluation

\section{INTRODUCTION}

There are many methods for the analysis of the city's economic strength in recent years, such as grey clustering analysis [1] [2], principal component analysis [3], and fuzzy comprehensive evaluation and so on. Neural Network is capable of automated learning and adaptation, meanwhile, processed large amount of parallel data and dis tributed information storage. However, It is difficult to evaluate importance and redundant of parameters [4]. Rough Set (RS) is new mathematic method for processing uncertain and incompatible data and imprecise question. Data attributes reduction and the minimum expression of knowledge can be solved after critical information kept, and then find implied knowledge and potential law [5]. RS and Neural Network combine advantages of two methods and make up for deficiencies [6] [7].

In this paper urban economy strength evaluation model which is based on RS and Neural Network puts forward to achieve effective evaluation on the city in Guangxi, and methods complement to urban economic.

\section{EVALUATION MODEL OF URBAN ECONOMY STRENGTH BASED ON RS AND NEURAL NETWORK}

\section{A. Construction of the Urban Economic Strength Evaluation Index System}

According to the basic principles of the scientific, guide, comparability, operability, referring to the related data and researcher on urban economy index system, The article has selected economic indexes which reflecting the level of economic

\author{
L.P. Zhang \\ College Mechanical and Control Engineering \\ Guilin University of Technology \\ China
}

development and establishing evaluation indexes system of urban economy strength[8]. Choosed 13 indexes as followed: (1) x1- Total Population(10000persons); (2) x2- Gross Domestic Product(100 million yuan); (3) x3-Primary Industry(100 million yuan); (4) $x 4-S e c o n d a r y$ Industry(100 million yuan); (5) x5-Industry(100 million yuan); (6) x6-Tertiary Industry (100 million yuan); (7) x7-Total Inves tment in Fixed As sets (100 million yuan); (8) x8-Total financial Revenue(100 million yuan); (9) x9Budgetary Revenue(100 million yuan); (10) x10- Budgetary Expenditure(100 million yuan); (11)xl1-Resident Savings Account(10000 yuan); (12)x12-total retail trade(100 million yuan).

\section{B. The Urban Economic Strength Evaluation Process}

Neural network has the advantages that distributed storage and parallel processing, automated learning and organization, generalization, fault tolerance and robustness; however, when large quantity of data is input, it causes huge neural network structure, long training time, and training failure. At the same time, it is decreased network generalization due to the using samples being contradiction and randomness inevitably. Moreover, accuracy of neural network itself also affects the accuracy of the evaluation results.

$\mathrm{RS}$ is a significant tool of analyzing incomplete, inaccurate information system, preprocessing input data of neural network by using RS, extracting the key ingredient as input of the network, reducing ruleset, simplifying the structure of the neural network, shorten the training time, improving the generalization ability, therefore,it can heighten evaluation reliability on the urban comprehensive economic strength. After decision table is reduced by $\mathrm{RS}$, which being a training sample set of neural networks [9]. RS-neural network model of urban economic strength evaluation process is shown in Fig 1:

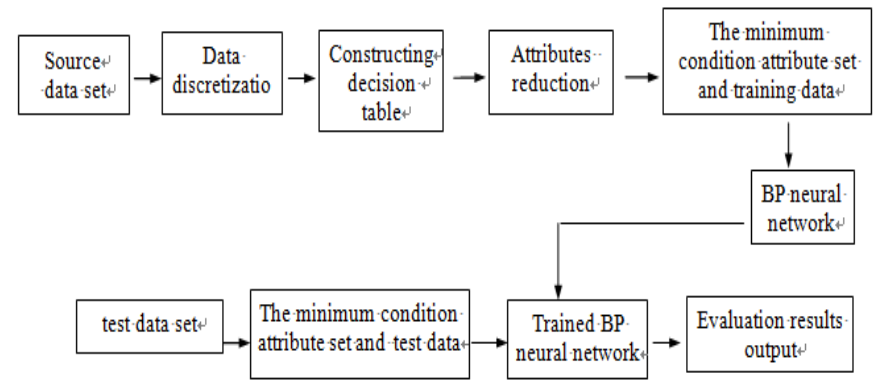

FIGURE I. EVALUTION PROCESS OF URBAN ECONOMY ST RENGT H BASED ON RS AND NEURAL NET WORK MODEL. 
Step1: Original data selected. Analyze data, select original sample set and form original decision table. The paper has selected 13 indexes as above.

Step2: Continuous data discretization. Bisection method can be used in this paper.

Step3: Form final decision table.

Step4: Determination of BP neural network evaluation model structure. It includes input layer, hidden layer and output layer, in hidden layer(n2) and input layer(n1):n2=2*n1+1. The economic evaluation grade divides into five grades: 1(lowest), 2(lower), 3(moderate); 4(higher); 5(highest),input sample expected value: lower $(1,0,0,0,0)$, low $(0,1,0,0,0)$, middle $(0$, $0,1,0,0)$,high $(0,0,0,1,0)$,higher $(0,0,0,0,1)$. Therefore, the number of nodes of the output layer is 5 .

Step5: Training of BP neural networks. Standardized data processing methods are as follows:

$$
\begin{gathered}
x_{i}^{\prime}=\left(x_{i}-x_{\min }\right) /\left(x_{\max }-x_{\min }\right) \\
x_{i}^{\prime}: \text { Standardized value; }{ }^{x_{i}} \text { : Target value; }{ }^{x_{\max } \text { :Maxium }}
\end{gathered}
$$
of evaluation indexes;

$X_{\text {min }}$ : Minimum of evaluation indexes; i: The number of evaluation indexes.

Step6: The urban economic strength evaluation process.

\section{APPLICATION}

The paper has choosed 13 cities in GuangXi as example to construct RS-neural network model(except for ChongZuo city) in the light of evaluation system above mentioned [10] [11]. The related data is shown in table1:

Urban economic strength decision table is shown in table2:

According to decision table definition:

$$
\begin{gathered}
\operatorname{Ind}(R)=\{(x, y) \in U \times U: r \in R: r(x)=r(y)\}(1) \\
A_{R}(X)=\frac{\left|R^{-} \cdot(X)\right|}{\left|R^{-}(X)\right|}
\end{gathered}
$$

In the type: $\mathrm{AR}(\mathrm{X})$ is approximation accuracy

$$
\mu \mathrm{RX}(\mathrm{x})=\frac{|X \cap R(X)|}{|R(X)|}
$$

In the type: $\mu \mathrm{RX}(\mathrm{x}) \in[0,1]$, value of $\mu \mathrm{RX}(\mathrm{x})$ is conditional probability, $\mathrm{x}$ is confidence level of $\mathrm{X}, \mathrm{RS}$ approaching as follow:

$$
\mathrm{R}-(\mathrm{X})=\{\mathrm{x} \in \mathrm{U}: \mu \mathrm{RX}(\mathrm{x})=1\} \quad \mathrm{R}-(\mathrm{x})=\{\mathrm{x} \in \mathrm{U}: \mu \mathrm{RX}(\mathrm{x})>0\}
$$

Let, suse MATLAB to realize RS- neural network evaluation model. Data in table3. Perform reduction with MATLAB RS function. The conclusion is realized [12]. $2,2,1 ; \ldots$

$\mathrm{C}=[2,3,1,3,2,3,1,2,2,2,2,2 ; 3,3,5,2,2,3,1,2,2,2,3,2 ; 3,2,4,2,2,2,1,1,2$,

$5,3,5,2,2,3,2,2,2,2,2,2 ; 5,2,4,2,2,2,1,2,2,2,3,2 ; 5,3,4,3,3,2,1,2,2,2,3$, $2 ; \ldots$
2,1,3,1,1,2,1,1,1,2,1,1;5,2,5,2,2,2,1,1,1,2,1,1;2,1,2,1,1,1,1,2,1,2,2, $1 ; \ldots$

$5,2,5,2,2,1,1,2,1,2,1,1 ; 5,5,4,5,3,5,5,5,4,5,5,5 ; 5,5,2,5,5,4,3,5,4,5,5$, $3 ; 4,4,3,4,4,4,3,4,3,5,4,3]$

$\mathrm{D}=[4 ; 3 ; 2 ; 2 ; 2 ; 2 ; 1 ; 2 ; 1 ; 2 ; 5 ; 4 ; 4]$;

$X=[C D] ; c=1: 13 ; d=14$;

$\mathrm{Y}=\mathrm{redu}(\mathrm{c}, \mathrm{d}, \mathrm{X})$

Operating results are, $\mathrm{Y}=1,2,3$, therefore, $\mathrm{x} 1, \mathrm{x} 2, \mathrm{x} 3$ can not be reduced, representing total population, Gross Domestic Product, Primary Industry. Three indexes are important data and factors reflecting city economic development level.

Three indexes extracted of five cities in table1.as learning sample data set, certain training parameters can be trained. In this paper, BP neural network uses LM algorithm, the maximum training step is 1000 , and 0.00001 squared error, hidden layer transfer function for tansig function, the output layer transformation function for logsig function. Research results are shown in Table3, it is obvious that BP neural network is absolutely capable of learning to identify these five samples, fitting $100 \%$, which indicates that the BP neural network has established between the complex nonlinear model evaluation criteria and evaluation level[13] [14]. In this case, trained the neural network has "simulated" and "memoried" "function" relationship between the input and output variables, it can be used to evaluate the urban economy.

According to the network acquired, the three indexes of remaining eight cities conduct the data standardization as the input of BP neural network, also evaluate BP network trained. In the light of how close the output value from the expected value, evaluation results obtained are shown in Table 4:

As can be seen from above network parameters and evaluation results, Evaluation model has corresponded with city economic development level and present condition. It is shown index system and RS-BP neural network evaluation model has a certain practical reference value.

\section{CONCLUSIONS}

This evaluation method has preprocessed neural network input data, eliminated redundant information, extracted key ingredients to reach simplifying the network structure; this can shorten the training time, to improve the recognition accuracy. Of course, the evaluation of the city economic strength is a complex system problems, it must be further study in the future on how to choose a more comprehensive index and a more efficient algorithm.

\section{ACKNOWLEDGEMENTS}

This work was financially supported by the Project of GuangXi Education Department Humanities and Social Science (LX2014480). 


\section{REFERENCES}

[1] Y. Shimoda,T. Fujii, T. Morikawa, M. Mizuno, Residential end use energy simulation at city scale. Building and Environment, 2004, 39(8), 959-967.

[2] S.L. Sun. Rescearch on the evaluation method of city economic level based on grey ystem[D]. Northeastern University,2012.

[3] H. Pang. Guangxi county economy comprehensive evaluation system based on principal component analysis and empirical analysis. Economic and Social Development, 2014,3(6)1-6.

[4] L.C. Jiao. Neural Network Application and Implementation (XiDian University press, 2006).

[5] Pawlak Z, Skowron A, Rough sets: some extensions, Information sciences, 2007(177) 28-40.

[6] R Yasdi. Combining rough sets learning and neural network learning method to deal with uncertain and imprecise information [J]. Neuron-computing, 2007(1). 61-64.
[7] X.M. Wang. Several Rough Models and the combanation of rough set and netural net work research [D].university of electronic science and technology of china, 2013.

[8] Z.Y. Huang, .Evaluation on Competitiveness ofChinese Medicine Industry Based on Rough Set and NeuralNetworkM odel Systems Engineering 2013, 31(6) 120-125.

[9] Chan C C,Grzymala Busse J W,Ziarko W P.Rough sets and current trends in computing//Proceedings of the 6th International Conference, RSCTC 2008.Akron,Ohio,USA,2008.

[10] Information on http://www.gxtj.gov.cn/.

[11] Information on http://www.cuew.com/.

[12] D.Y. Xue,Y.Q. Chen,MATLAB mathematical problem solving 2004 , 347-356.

[13] Y.Y. Yao, Y. Zhao.Discernibility matrix simplication for constructing contribute reducts [J]. Information Sciences, 2009, 179(7) 867-882.

[14] C.J. Yang, H. GE, Z.S. Wang. Overview of attribute reduction based on rough set [J]. Application Research of Computers, 2012,29(1) 16-20.

TABLE I. CIT Y ECONOMIC DEVELOPMENT LEVEL INDEX VALUE.

\begin{tabular}{|c|c|c|c|c|c|c|c|c|}
\hline Iities & & & & & & & & \\
& & $\mathrm{x} 1$ & $\mathrm{x} 3$ & $\ldots$ & $\mathrm{x} 10$ & $\mathrm{x} 11$ & $\mathrm{x} 12$ & Evaluation grade \\
\hline WuZhou & 34.01 & 62.11 & 1.31 & $\ldots$ & 8.86 & 47.66 & 29.16 & higher \\
\hline BeiHai & 52.90 & 82.26 & 18.07 & $\ldots$ & 9.54 & 55.8 & 30.51 & moderate \\
\hline FangChengGang & 47.44 & 45.17 & 11.77 & $\ldots$ & 4.90 & 26.86 & 17.03 & lower \\
\hline QinZhou & 119.34 & 75.16 & 35.38 & $\ldots$ & 7.31 & 34.47 & 31.20 & lower \\
\hline GuiGang & 167.58 & 47.82 & 14.48 & $\ldots$ & 6.50 & 44.27 & 21.56 & lower \\
\hline YuLin & 89.70 & 57.04 & 11.57 & $\ldots$ & 7.65 & 79.56 & 39.96 & lower \\
\hline BaiSe & 32.71 & 29.86 & 5.83 & $\ldots$ & 2.71 & 21.30 & 8.67 & lowest \\
\hline HeZhou & 90.37 & 50.88 & 18.21 & $\ldots$ & 2.88 & 23.02 & 16.16 & lower \\
\hline HeChi & 31.23 & 26.44 & 3.75 & $\ldots$ & 2.31 & 25.99 & 13.74 & lowest \\
\hline LaiBin & 96.67 & 50.96 & 16.35 & $\ldots$ & 4.05 & 13.92 & 8.77 & lower \\
\hline NanNing & 140.39 & 269.06 & 10.75 & $\ldots$ & 27.86 & 290.64 & 159.76 & highest \\
\hline LiuZhou & 94.38 & 179.94 & 3.80 & $\ldots$ & 19.71 & 166.88 & 74.63 & higher \\
\hline GuiLin & 69.09 & 127.82 & 6.40 & $\ldots$ & 16.37 & 138.15 & 65.98 & higher \\
\hline
\end{tabular}

T ABLE II. CITY ECONOMIC ST RENGTH DECISION T ABLE.

\begin{tabular}{|c|c|c|c|c|c|c|c|c|}
\hline \multirow{2}{*}{ U(cities) } & \multicolumn{7}{|c|}{ C(condition attributes) } & \multirow{2}{*}{$\begin{array}{c}\text { D(decision } \\
\text { attributes) }\end{array}$} \\
\cline { 2 - 9 } & $\mathrm{x} 1$ & $\mathrm{x} 2$ & $\mathrm{x} 3$ & $\ldots$ & $\mathrm{x} 10$ & $\mathrm{x} 11$ & $\mathrm{x} 12$ & 4 \\
\hline WuZhou & 2 & 3 & 1 & $\ldots$ & 2 & 2 & 2 & 3 \\
\hline BeiHai & 3 & 3 & 5 & $\ldots$ & 2 & 3 & 2 & 2 \\
\hline FangChengGang & 3 & 2 & 4 & $\ldots$ & 2 & 2 & 1 & 2 \\
\hline QinZhou & 5 & 3 & 5 & $\ldots$ & 2 & 2 & 2 & 2 \\
\hline GuiGang & 5 & 2 & 4 & $\ldots$ & 2 & 3 & 2 & 2 \\
\hline YuLin & 5 & 3 & 4 & $\ldots$ & 2 & 3 & 2 & 1 \\
\hline BaiSe & 2 & 1 & 3 & $\ldots$ & 2 & 1 & 1 & 2 \\
\hline HeZhou & 5 & 2 & 5 & $\ldots$ & 2 & 1 & 1 & 1 \\
\hline HeChi & 2 & 1 & 2 & $\ldots$ & 2 & 2 & 1 & 2 \\
\hline LaiBin & 5 & 2 & 5 & $\ldots$ & 2 & 1 & 1 & 5 \\
\hline NanNing & 5 & 5 & 4 & $\ldots$ & 5 & 5 & 5 & 4 \\
\hline LiuZhou & 5 & 5 & 2 & $\ldots$ & 5 & 5 & 3 & 4 \\
\hline GuiLin & 4 & 4 & 3 & $\ldots$ & 5 & 4 & 3 & 2 \\
\hline
\end{tabular}


TABLE III. BP NEURAL NETWORK LEARNING RESULTS.

\begin{tabular}{|c|c|c|c|c|c|c|c|c|c|c|}
\hline grades & \multicolumn{6}{|c|}{ Desired out put } & \multicolumn{5}{|c|}{ BP net work actual output } \\
\hline 1 & 1 & 0 & 0 & 0 & 0 & 0.9876 & 0.0000 & 0.0023 & 0.0000 & 0.0000 \\
\hline 2 & 0 & 1 & 0 & 0 & 0 & 0.0000 & 0.9986 & 0.0000 & 0.0012 & 0.0001 \\
\hline 3 & 0 & 0 & 1 & 0 & 0 & 0.0000 & 0.0000 & 0.9999 & 0.0003 & 0.0000 \\
\hline 4 & 0 & 0 & 0 & 1 & 0 & 0.0002 & 0.0000 & 0.0003 & 0.9989 & 0.0013 \\
\hline 5 & 0 & 0 & 0 & 0 & 1 & 0.0000 & 0.0007 & 0.0000 & 0.0000 & 0.9992 \\
\hline
\end{tabular}

T ABLE IV. CITY ECONOMIC EVALUATION RESULTS.

\begin{tabular}{|c|c|c|c|c|c|c|}
\hline Cities & \multicolumn{5}{|c|}{ Network out put } & Evaluation results \\
\hline WuZhou & 0.0146 & 0.0000 & 0.0000 & 0.9625 & 0.0001 & 4(higher) \\
\hline FangChengGang & 0.0000 & 0.9795 & 0.0000 & 0.0006 & 0.0000 & 2(lower) \\
\hline QinZhou & 0.0000 & 0.9980 & 0.0012 & 0.0000 & 0.0000 & 2 (lower) \\
\hline YuLin & 0.0000 & 0.0002 & 0.9999 & 0.0000 & 0.0000 & 2 (lower) \\
\hline BaiSe & 0.9998 & 0.0000 & 0.0027 & 0.0000 & 0.0000 & 1(lowest) \\
\hline HeZhou & 0.0008 & 0.9648 & 0.0000 & 0.0000 & 0.0000 & 2(lower) \\
\hline LaiBin & 0.0000 & 0.9999 & 0.0034 & 0.0000 & 0.0000 & 2(lower) \\
\hline LiuZhou & 0.0000 & 0.0000 & 0.0000 & 0.9887 & 0.0019 & 4(higher) \\
\hline
\end{tabular}

